\title{
Actitudes lingüísticas en una situación de contacto de lenguas
}

\author{
María Victoria NAVAS SÁNCHEZ-ÉLEZ \\ Universidad Complutense de Madrid \\ Centro de Linguística da Universidade de Lisboa \\ mvnavas@ucm.es
}

\begin{abstract}
RESUMEN
Se presentan los resultados de una encuesta sobre actitudes lingüísticas llevada a cabo en Barrancos (Beja, Portugal), comunidad donde se hablan tres lenguas, portugués, español y barranqueño. Partiendo de unos determinados itens se pregunta a los miembros de la comunidad cuál es su actitud ante cada una de las lenguas habladas. El análisis refleja los resultados totales pero también por sexos, niveles de escolarización y edades.
\end{abstract}

Palabras clave: Barranqueño, actitudes lingüísticas, frontera lingüística.

[Recibido, diciembre 2013; aprobado, mayo 2014]

Linguistic attitudes in a situation of contact of languages

\begin{abstract}
The results of a survey on linguistic attitudes carried out in Barrancos (Beja, Portugal), a community where three languages are spoken, Portuguese, Spanish and Barranqueño are presented. Starting from a certain itens are asked community members what their attitude to each of the languages spoken. The analysis reflects the overall results but also by gender, education levels and ages.
\end{abstract}

Key words: Barranqueño, language attitudes, language border. 


\section{Presentación}

Forma parte del protocolo de las investigaciones sociolingüísticas medir la valoración subjetiva de los usos lingüísticos de una determinada comunidad y para ello se han venido utilizando diferentes métodos (Silva Corvalán 1988: 41 y ss.). En nuestro caso ${ }^{1}$ nos interesaba estudiar las actitudes lingüísticas de los habitantes de Barrancos (Portugal) en lo que se refiere a las lenguas en contacto que existen en la población -el portugués y el español- y la lengua producto y suma de las dos -el barranqueño- para conocer el nivel de prestigio que las tres lenguas -la española, la barranqueña y la portuguesa- tiene entre los hablantes de dicha comunidad.

Barrancos es una villa portuguesa de aproximadamente 2000 habitantes, que se encuentra al suroeste del distrito de Beja (Portugal) en la frontera con el suroeste de la provincia de Badajoz y el noroeste de la provincia de Huelva, en un espacio geográfico donde el río Guadiana no es frontera entre España y Portugal y donde el paso del mencionado río es fácil debido a su escaso caudal en la zona. Este motivo y otras circunstancias de orden político y social -línea defensiva, repoblaciones españolas a lo largo del tiempo, contrabando, aislamiento- ha permitido la creación de una lengua mixta, fruto del contacto del portugués y del español meridionales (Navas 2011), de manera que en la villa citada se puede oír hablar portugués, barranqueño y español. De hecho, en una encuesta facilitada por el Prof. Víctor Adragão (1976) referida a su trabajo realizado en la comunidad en los años 70 dice el sujeto grabado: "Nós aqui falamos de varias maneras. Por exemplo, de "a farinha" dizemos [a fa 'rin $\alpha$ ] quando falamos portugués; [a a 'rina] quando falamos barranquenho; e [la ha "rina] quando falamos espanhol”. Es Barrancos, pues, un pueblo que ha vivido a caballo entre dos países buscando su propia naturaleza, su idiosincrasia y su aspecto distintivo; para ello se ha servido de la lengua como sistema lingüístico específico, resultado, ya se ha dicho, del contacto del portugués y del castellano. Pero debido a sus circunstancias territoriales, a su forma de concebir su cultura, ha logrado encontrar su personalidad y su medio de expresión lingüística.

En diversos estudios hemos descrito algunas de las características lingüísticas del barranqueño de entre las que destacamos las más significativas desde el punto de vista fonológico, como son la aspiración de la sibilante en posición final de sílaba o de palabra: [i te

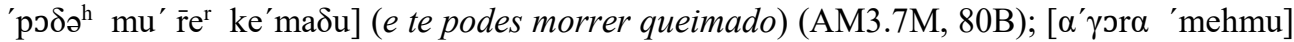
(agora mesmo) (AM3.7M, 80B); o la desaparición de $<\mathrm{l}>\mathrm{o}<\mathrm{r}>$ finales: [ $\alpha \mathrm{h}$ pə'so $\alpha$ 'majh

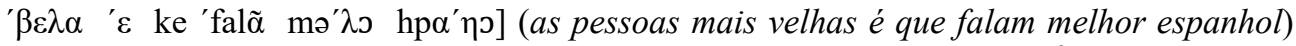

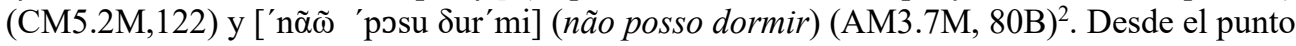
de vista morfosintáctico señalamos alguna marca de género en femenino como a sangue (port. "o sangue", esp. "la sangre"); o peral (port. "a pereira", esp. "el peral"); ausencia de concordancia de número en el $\mathrm{SN}$ : as pernas eram mais lista (esp. "las piernas eran más rápidas"); o la colocación del pronombre átono según la norma española actual y la antigua

\footnotetext{
${ }^{1}$ Texto leído en el XXVII Congreso Internacional de Lingüística y Filología Románicas celebrado en Nancy (15-20 julio, 2013).

${ }_{2}^{2}$ Recordemos, en primer lugar, que estos fenómenos no son únicos del barranqueño pues los podemos encontrar en otras zonas fronterizas hispano-portuguesas y también uruguayo-brasileñas (Elizaincín 1992) en diferente grado o nivel de implantación; en segundo lugar, que como el barranqueño es una lengua solo oral la ortografía aquí transcrita es de nuestra responsabilidad.
} 
portuguesa: nos casamentos se costuma cantar ao espanhol ou sevilhanas ou alentejano (esp. "en las bodas se suele cantar en español o por sevillanas o en alentejano"). Además de ciertas partículas y léxico importados del español como hasta (port. "até", esp. "hasta”), bueno en inicio de frase (port. "então", esp. "bueno"); párpado (port. "pálpebra”, esp. "párpado"); hipo (port. "soluço", esp. "hipo"). No podemos dejar de señalar el importante corpus de tradición oral que pervive conservado en español, como canciones, romances, anécdotas o cuentos.

En definitiva queríamos observar si había algún prejuicio lingüístico para cada una de las referidas lenguas, si se manifestaba algún tipo de lealtad para alguna de ellas o, por último, si se reafirmaba su identidad lingüística. Además, pretendíamos saber si dicha actitud estaba condicionada por factores tales como la edad, el sexo y el grado o no de escolarización. Dentro de una amplia recolección de datos con un cuestionario cerrado acompañado de una serie de entrevistas semidirigidas, esencialmente realizadas entre 1989 y 1990, incluimos esta prueba formal como complemento para poder observar la conciencia lingüística de los barranqueños sobre las lenguas habladas en su comunidad.

\section{Metodología}

Utilizamos una de las técnicas más frecuentemente para estos casos, el diferencial semántico, para evaluar la actitud de los sujetos, aunque somos conscientes de las dificultades que implica la interpretación de estos resultados pues desconocemos el impulso que lleva al sujeto a inclinarse por una u otra opción. Presentamos seis adjetivos antónimos que los informantes debían situar entre una de siete posibilidades a partir de su propia percepción.

\section{Itens analizados ${ }^{3}$}

\begin{tabular}{|c|c|c|c|c|c|c|c|c|c|}
\hline Valoración & $\mathbf{7}$ & $\mathbf{6}$ & $\mathbf{5}$ & & $\mathbf{4}$ & $\mathbf{3}$ & $\mathbf{2}$ & $\mathbf{1}$ & Valoración \\
\hline bonito & --- & --- & --- & & --- & --- & --- & --- & feo \\
\hline agradable & --- & --- & --- & & --- & --- & --- & --- & desagradable \\
\hline alegre & --- & --- & --- & & --- & --- & --- & --- & triste \\
\hline rápido & --- & --- & --- & & --- & --- & --- & --- & lento \\
\hline fácil & --- & --- & --- & & --- & --- & --- & --- & difícil \\
\hline ventajoso & --- & --- & --- & & --- & --- & --- & --- & no ventajoso \\
\hline
\end{tabular}

Para el presente trabajo hemos seleccionado exclusivamente las valoraciones máximas, es decir, aquellas a las que los informantes otorgaron el valor de siete. En estudios posteriores podríamos ponderar nuestras conclusiones con un análisis más complejo e incluso compararlo con los resultados de una futura encuesta ${ }^{4}$, cuyo objetivo sería observar las posibles fluctuaciones en estos últimos años.

\footnotetext{
${ }^{3}$ La entrevista y el cuestionario se realizaron en portugués. Agradezco a Jesús Bermejo Bermejo la elaboración de todos los cuadros matemáticos que han servido de base para este estudio.

${ }^{4}$ Victor Correia prepara su tesis doctoral en la Universidad de Évora, Proposta de uma Convenção Ortográfica de uma língua de Contacto no século XXI: Aplicação ao Barranquenho, dirigida al alimón por la Dra. Gonçalves y la que suscribe, donde el investigador ha incluido también unas entrevistas sobre actitudes lingüísticas de los hablantes de barranqueño. Dicho trabajo sin duda servirá para hacer un cronoestudio del fenómeno.
} 
Los sujetos ${ }^{5}$ entrevistados para este apartado, que no corresponden exactamente a los analizados en el trabajo estadístico mencionado (Navas 2011), fueron 43 personas, 21 mujeres y 22 hombres. Se dividieron los sujetos por su grado de escolarización: 7 no escolarizados, 21 con estudios primarios, 15 con estudios secundarios. Así como por grupos de edades: de 12 a 25 años, 14; entre 25 y 45 años, 13, y mayores de 45 años, 16.

\section{Resultados}

\subsection{Resultados totales}

\begin{tabular}{|c|c|c|c|c|}
\hline \multicolumn{5}{|c|}{ Porcentajes } \\
\hline & Valoración & Barranqueño & Español & Portugués \\
\hline \multirow{3}{*}{ Resultados totales } & bonito & $48,84 \%$ & $62,79 \%$ & $62,79 \%$ \\
\cline { 2 - 5 } & agradable & $32,56 \%$ & $46,51 \%$ & $46,51 \%$ \\
\cline { 2 - 5 } & alegre & $46,51 \%$ & $76,74 \%$ & $30,23 \%$ \\
\cline { 2 - 5 } & rápido & $55,81 \%$ & $55,81 \%$ & $23,26 \%$ \\
\cline { 2 - 5 } & fácil & $23,26 \%$ & $34,88 \%$ & $13,95 \%$ \\
\cline { 2 - 5 } & ventajoso & $25,58 \%$ & $34,88 \%$ & $46,51 \%$ \\
\hline
\end{tabular}

A partir del Cuadro $\mathrm{n}^{\circ} 1$ se deduce que los hablantes de barranqueño piensan que el español y el portugués son más "bonito"s $(62,79 \%)$ que su propia lengua $(48,84 \%)$ aunque tan "rápido" como el español $(55,81 \%)$. Que el español es significativamente el más "alegre" (76,74\%) pues el barranqueño lo es en un $46,51 \%$ y el portugués en 30,23\%. Mientras que el portugués es, como ya se ha dicho, "bonito" pero el menos "rápido" $(23,26 \%)$ y "fácil" de los tres $(13,95 \%)$ y aporta su conocimiento más ventajas $(46,51 \%)$ que el de las otras dos lenguas de la comunidad. En resumen, para los hablantes de barranqueño su lengua es rápida -tanto como el español- pero menos bonita que el español y que el portugués. Mientras que el español es alegre, el portugués es poco "rápido" y muy poco "fácil” pero "ventajoso", siendo, pues, la única actitud positiva del barranqueño su rapidez. En general, los entrevistados valoran más y mejor el español que el barranqueño. En lo que se refiere al portugués frente al barranqueño, los informantes piensan que la primera es más bonita, más agradable y más ventajosa que la segunda; mientras que esta segunda, el barranqueño, es más alegre, más rápida y más fácil que el portugués. La apreciación de "alegre" para el español veremos, por cierto, que va a ser una constante en todas las respuestas de todos los grupos en un porcentaje siempre superior al $50 \%$.

\footnotetext{
${ }^{5}$ Para tener noticia de las personas entrevistadas, véase Navas (1011).
} 


\subsection{Resultados por sexos}

\begin{tabular}{|c|c|c|c|c|c|}
\hline \multicolumn{6}{|c|}{ Cuadro n' 2} \\
\hline & & Valoración & Barranqueño & Español & Portugués \\
\hline \multirow{12}{*}{$\begin{array}{c}\text { Por } \\
\text { sexos }\end{array}$} & \multirow{6}{*}{ Hombres } & bonito & $59,09 \%$ & $68,18 \%$ & $68,18 \%$ \\
\hline & & agradable & $40,91 \%$ & $54,55 \%$ & $59,09 \%$ \\
\hline & & alegre & $50,00 \%$ & $81,82 \%$ & $31,82 \%$ \\
\hline & & rápido & $63,64 \%$ & $54,55 \%$ & $27,27 \%$ \\
\hline & & fácil & $27,27 \%$ & $50,00 \%$ & $9,09 \%$ \\
\hline & & ventajoso & $31,82 \%$ & $36,36 \%$ & $50,00 \%$ \\
\hline & \multirow{6}{*}{ Mujeres } & bonito & $38,10 \%$ & $57,14 \%$ & $57,14 \%$ \\
\hline & & agradable & $23,81 \%$ & $38,10 \%$ & $33,33 \%$ \\
\hline & & alegre & $42,86 \%$ & $71,43 \%$ & $28,57 \%$ \\
\hline & & rápido & $47,62 \%$ & $57,14 \%$ & $19,05 \%$ \\
\hline & & fácil & $19,05 \%$ & $19,05 \%$ & $19,05 \%$ \\
\hline & & ventajoso & $19,05 \%$ & $33,33 \%$ & $42,86 \%$ \\
\hline
\end{tabular}

Si nos centramos en la diferencia de actitudes lingüísticas entre hombres y mujeres, verificaremos que para los hombres, en orden decreciente, el barranqueño es "rápido" (63,64\%), "bonito" (59,09\%) y "alegre" (50\%). Mientras que el español para este grupo es "alegre" (81,82\%), "bonito" (68,18\%), "agradable", "rápido" (54,55\%) y "fácil” (50,00\%). Finalmente, el portugués es tan "bonito" como el español $(68,18 \%)$, "agradable" (59,09\%) y "ventajoso" (50\%) pero muy poco "fácil" en un elevado porcentaje $(9,09 \%)$.

Las respuestas de las mujeres, con muchos más matices, muestran, en primer lugar, que las puntuaciones para el barranqueño son bastante inferiores a las dadas a las otras dos lenguas, pues nunca el porcentaje es superior al 50\%. En segundo lugar, el español es "alegre" (71,43\%), al mismo tiempo que "bonito" y "rápido" (57,14\%). Mientras que el portugués es "bonito" (57,14\%) y poco "rápido" (19,05\%). Una actitud compartida, por otra parte, para todas las lenguas es lo poco fácil que piensan que son las tres $(19,05 \%)$.

Contrastando las respuestas de unos y otras verificamos que estas últimas manifiestan una actitud de menor valoración para con el barranqueño pues, además, ellas entienden que la citada lengua es más difícil (en un 8,22\%) y tiene menos ventajas (en 12,77\%) que para sus compañeros de encuesta. En cuanto al español la diferencia más destacada entre unos y otras es que estas lo consideran mucho menos "fácil" y menos "rápido" que los hombres. Respecto al portugués los datos indican que las mujeres entienden que esta lengua es un $3,25 \%$ menos alegre, un 8,22\% menos "fácil" y menos rápida que para los hombres. En resumen, estamos ante una valoración más positiva para el barranqueño, el español y el portugués por parte de los hombres que de las mujeres. 


\subsection{Resultados por niveles de escolarización}

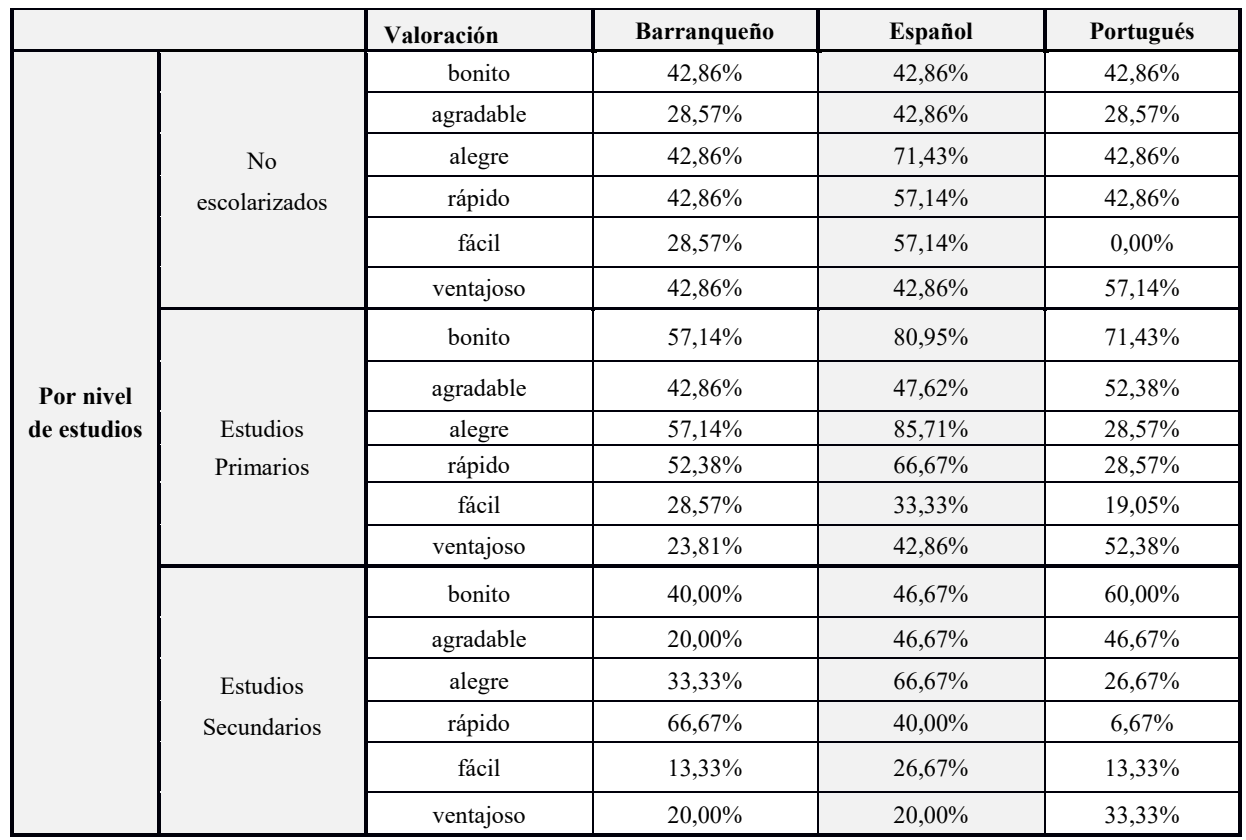

Cuadro no 3

En lo referente al Cuadro $\mathrm{n}^{\mathrm{o}} 3$, al pronunciarnos sobre las valoraciones del grupo de los no escolarizados, deducimos, en primer lugar, que no hay actitud positiva para el barranqueño. Mientras sí consideran el español "alegre" (71,43\%), "rápido" y "fácil" (57,14\%), notan que el portugués es muy poco "fácil", es decir, difícil en un (100\%) aunque destacan, no obstante, la ventaja de saberlo (57,14\%), pues consideran, tal vez, que solo con el barranqueño no se obtiene promoción social.

El grupo de informantes con estudios primarios es el que mejor valora el barranqueño, pues lo considera "bonito" (57,14\%), "alegre" (57,14\%), "rápido" (52,38\%) y "agradable" $(42,86 \%)$. El español, por su parte, es asimismo favorablemente valorado como "alegre" $(85,71 \%)$, "bonito" $(80,95 \%)$ y "rápido" $(66,74 \%)$. En cuanto al portugués destacan que es "bonito" (71,43\%), "agradable" y "ventajoso" (52,38\%) aunque poco "fácil” (19,05\%).

Las personas con estudios secundarios hacen una valoración poco positiva del barranqueño. Quizá porque tienen una escolarización en portugués, la lengua del estado al que pertenece Barrancos, y esa formación les ha mostrado que el barranqueño no les sirve para promocionarse en el estado portugués. Es en la franja de los sujetos con estudios primarios, frente a los otros dos grupos, donde el barranqueño se encuentra mejor valorado. Y, curiosamente, son estos mismos los que tienen, cuantitativamente hablando, mayor estima hacia el español y hacia el portugués. Los sujetos con estudios secundarios entienden que el barranqueño es "rápido" $(66,67 \%)$. Y si tenemos en cuenta a los que lo valoran con 6, también es "bonito", "agradable" y "alegre" aunque muy poco "fácil" (13,33\%). El español es sobre todo "alegre" y el portugués "bonito" $(60,00 \%)$ pero muy poco "fácil" $(13,33 \%)$. 
En conclusión, hemos de decir que para los no escolarizados el barranqueño es considerado fácil $(28,57 \%$ con valoración 7 y $71,43 \%$ con valoración 6$)$ y el portugués, muy poco fácil $(0,00 \%$ con valoración 7 y solo $42,86 \%$ con valoración 6$)$. Para los informantes con estudios primarios el barranqueño es poco fácil $(28,57 \%)$ y el portugués muy poco fácil $(19,05 \%)$. Para los entrevistados con estudios secundarios el barranqueño es muy poco fácil $(13,33 \%) \mathrm{y}$, curiosamente, el portugués es también muy poco fácil $(13,33 \%)$.

\subsection{Resultados por edades}

\begin{tabular}{|c|c|c|c|c|c|}
\hline & & Valoración & Barranqueño & Español & Portugués \\
\hline \multirow{18}{*}{$\begin{array}{c}\text { Por tramos } \\
\text { de edad }\end{array}$} & \multirow{6}{*}{$\begin{array}{c}12 \text { a } 25 \\
\text { años }\end{array}$} & bonito & $57,14 \%$ & $57,14 \%$ & $57,14 \%$ \\
\hline & & agradable & $21,43 \%$ & $35,71 \%$ & $21,43 \%$ \\
\hline & & alegre & $35,71 \%$ & $64,29 \%$ & $14,29 \%$ \\
\hline & & rápido & $57,14 \%$ & $57,14 \%$ & $0,00 \%$ \\
\hline & & fácil & $21,43 \%$ & $14,29 \%$ & $0,00 \%$ \\
\hline & & ventajoso & $7,14 \%$ & $14,29 \%$ & $35,71 \%$ \\
\hline & \multirow{6}{*}{$\begin{array}{c}25 \text { a } 45 \\
\text { años }\end{array}$} & bonito & $46,15 \%$ & $76,92 \%$ & $84,62 \%$ \\
\hline & & agradable & $38,46 \%$ & $46,15 \%$ & $84,62 \%$ \\
\hline & & alegre & $61,54 \%$ & $92,31 \%$ & $46,15 \%$ \\
\hline & & rápido & $69,23 \%$ & $61,54 \%$ & $23,08 \%$ \\
\hline & & fácil & $30,77 \%$ & $46,15 \%$ & $30,77 \%$ \\
\hline & & ventajoso & $23,08 \%$ & $38,46 \%$ & $46,15 \%$ \\
\hline & \multirow{6}{*}{$\begin{array}{l}\text { Mayores de } \\
45 \text { años }\end{array}$} & bonito & $43,75 \%$ & $56,25 \%$ & $50,00 \%$ \\
\hline & & agradable & $37,50 \%$ & $56,25 \%$ & $37,50 \%$ \\
\hline & & alegre & $43,75 \%$ & $75,00 \%$ & $31,25 \%$ \\
\hline & & rápido & $43,75 \%$ & $50,00 \%$ & $43,75 \%$ \\
\hline & & fácil & $18,75 \%$ & $43,75 \%$ & $12,50 \%$ \\
\hline & & ventajoso & $43,75 \%$ & $50,00 \%$ & $56,25 \%$ \\
\hline
\end{tabular}

En lo que se refiere a la franja de edad de los jóvenes entre 12 a 25 años, destacamos, en primer lugar, que para ellos tanto el barranqueño, como el español o el portugués tienen idéntico rango para "bonito" (57,14\%). Pero así como las dos primeras lenguas, barranqueño y español, presentan el mismo porcentaje para "rápido" $(57,14 \%)$ el portugués es muy poco "rápido" y muy poco "fácil" en el $(100 \%)$ de las respuestas. Además, para este grupo el barranqueño es la lengua que menos ventajas tiene de las tres $(7,14 \%)$. La idea de que el portugués sea poco "fácil" para este conjunto de personas es comprensible dado que se trata de un grupo en edad escolar, que tiene que enfrentarse al aprendizaje normativo del portugués y en portugués.

Los sujetos de entre 25 y 45 años consideran que el barranqueño es sobre todo "rápido" $(69,23 \%)$ y "alegre" $(61,54 \%)$ pero ni tiene ventajas conocerlo $(23,08 \%)$ ni es "fácil" (30,77\%). Mientras, el español es especialmente "alegre" (92,31\%), "bonito" (76,92\%) y "rápido" (61,54\%), y el portugués es asimismo bien valorado, como "bonito" y "agradable" $(84,62 \%)$, pero poco "fácil” (30,77\%).

Los entrevistados mayores de 45 años, responden que el barranqueño es difícil ("fácil" para el 18,75\%) aunque más difícil es para este grupo el portugués (“fácil para el 12,50\%). Respecto del español estos hablantes presentan una gama de entradas favoritas: es sobre 
todo "alegre" (75\%), "bonito" y "agradable" (56,25\%), "rápido" y "ventajoso" (50,00\%). En lo que se refiere al portugués destacan la ventaja indudable de conocerlo $(56,25 \%)$ y su belleza ("bonito" por el 50,0\%).

Finalmente, hemos de señalar que, para los informantes de 12 a 25 años, a la vista de los porcentajes que muestra el Cuadro $n^{\circ} 4$, el barranqueño es poco "fácil" $(21,43 \%)$ pero es aún menos fácil el portugués $(0,00 \%)$. Para los informantes de 25 a 45 años tanto el barranqueño como el portugués son poco "fáciles" en el mismo porcentaje $(30,77 \%)$. En cuanto a los entrevistados mayores de 45 años consideran que el barranqueño es "fácil" (18,75\%) mientras que el portugués es muy poco "fácil” $(12,50 \%)$.

\section{Mirando al futuro}

Recordamos que todos los datos analizados en este trabajo proceden de una encuesta realizada hace varios años. Desde entonces, muchas cosas han ocurrido en Barrancos y en el barranqueño. A la luz de la estima que tienen los habitantes de dicha villa por su propia lengua nos parece que los datos de la encuesta que hemos analizado, muestran unas valoraciones que pueden haber cambiado sustancialmente. Es frecuente oír en Barrancos esta frase: "Nós não somos nem portugueses, nem espanhóis, somos barranquenhos". Es decir, en la comunidad barranqueña hay personas trilingües y bilingües, y la preocupación por conservar el habla barranqueña ha sido constante. Se desprende, pues, de todo lo anteriormente dicho hasta ahora que estamos ante un pueblo fronterizo entre España y Portugal con unas características que le convierten en único ${ }^{6}$. Si en nuestra opinión, ha existido siempre en la comunidad de Barrancos la conciencia de su especificidad, nos parece que esta se hizo más evidente sobre todo a partir de su lucha por mantener sus costumbres frente al poder oficial. Nos estamos refiriendo, concretamente, a los festejos taurinos como ejemplo paradigmático que fueron el motor de arranque y palanca de la lengua como soporte de su identidad, como resorte del despertar lingüístico de la comunidad en defensa de la oficialidad de su lengua, a inicios del presente siglo.

Veamos los hechos. En Portugal, desde 1928, no se matan los toros en la plaza en el transcurso de la lidia, mientras que en Barrancos el toro muere en el ruedo, a la manera española. Durante muchos años esta práctica barranqueña fue perseguida por la policía. Ello dio lugar, como se comprende, a muchos problemas, denuncias y multas al consistorio de Barrancos por desacato a la ley; complicaciones, dicho sea de paso, que se iban resolviendo sin mayor recorrido y archivando año tras año. Así hasta que, finalmente, en julio de 2002, la Inspección General de las Actividades Culturales, aprobó, después de haber sido modificada la ley en el Parlamento luso, esta práctica taurina en Barrancos, basándose en la tradición de los citados festejos. Esta reivindicación de su pasado taurino, por parte de los habitantes de Barrancos, tuvo una importante consecuencia pues logró que la población se aglutinase en defensa de su identidad (Franco 2000).

Antes de la aprobación de dicha ley los barranqueños manifestaron de diferentes formas su protesta y su reivindicación. Por ello, para exteriorizar su desacuerdo, surgieron diversas iniciativas -carteles, pancartas, camisetas, eslóganes- que los barranqueños precisaban

\footnotetext{
${ }^{6}$ Por ejemplo, SOS, el Grupo de Teatro de Barrancos, representa sus obras en barranqueño con expresiones en español con gran regocijo por parte del público.
} 
redactar, para indicar, por un lado, su protesta y su malestar, y, por otro, para mostrar al resto de los portugueses su peculiaridad, en las diversas ocasiones en que se presentaron frente al Parlamento portugués en Lisboa. En ese momento los barranqueños confirmaron lo que ya se sabía, que no existía un código escrito para transcribir sus protestas en su propia lengua. Tuvieron, entonces, que recurrir a una norma fonético-ortográfica, propuesta en los años treinta del siglo pasado, para uso de lingüistas, por el filólogo Leite de Vasconcelos (1955), norma que no cubría sus necesidades ortográficas. Este fue el motivo para que se dieran pasos en el sentido de que la comunidad exigiera una norma ortográfica, una gramática y un diccionario de su misma lengua ${ }^{7}$. Por ello, desde hace años los diferentes equipos del Ayuntamiento barranqueño luchan para que la lengua tenga una declaración oficial del mismo rango que la lengua mirandesa, que posee un estatuto específico de lengua cooficial en Portugal en la región de Miranda do Douro, desde 1999.

Así, en 2002, surgió una iniciativa minoritaria, Mubimênto du Pobu Barranquenhu di Barrancû en Purtugá (MPB), formado por siete personas, con el objetivo de "encontrar as melhores soluções prácticas para defender os interesses do povo do concelho de Barrancos. Fomentar e divulgar o ensino da língua Barranquenha (...); tornar o Barranquenho como língua oficial em todo o concelho de Barrancos, a par do Português (...). Estamos comprometidos com a autonomia de Barrancos dentro de um Portugal multicultural e regional".

Pero ha sido sobre todo recientemente cuando se consiguió, de acuerdo con la Convención para la Salvaguarda del Patrimonio Cultural Inmaterial de la UNESCO, que el Municipio de Barrancos, mediante aprobación por unanimidad de la Asamblea Municipal, en 2008, decidiese clasificar el dialecto barranqueño como Patrimonio Cultural Inmaterial de Interés Municipal.

Pero, además, el concejo quiere que la lengua barranqueña tenga un reconocimiento mayor, y pretende, desea y lucha para que esta se convierta en Patrimonio Lingüístico Nacional, pues para esta villa rayana, en palabras de su edil a la Agencia Lusa: "Queremos preservar e valorizar o barranquenho que é um dialecto único e um elemento de valor incalculável e indispensável para conhecer e entender a identidade da terra e das gentes de Barrancos"s.

Además, siguiendo la pauta de promocionar su idioma, el municipio de Barrancos, con los correspondientes españoles de Cedillo y Herrera de Alcántara, localidades de la provincia de Cáceres, donde se hablan variedades del portugués, el Centro de Lingüística de la Universidad de Lisboa, y las Universidades de Évora y de Extremadura, firmaron un protocolo con el objetivo de poner en marcha un proyecto para "estudar, preservar e valorizar as falas destes povos", que arrancó en ese mismo año de $2008^{9}$. Según la Vicepresidenta del Municipio, Isabel Sabino, el proyecto va a "actualizar os estudos sobre a história, o vocabulário e a gramática do barranquenho" porque, explicó la referida política, "o objectivo é conseguir que o barranquenho seja reconhecido oficialmente como um dialecto,

\footnotetext{
${ }^{7}$ Son varias las personas que al querer escribir la lengua hablada han optado por una u otra grafía casi siempre próxima a la norma propuesta por Leite de Vasconcelos, como el poeta popular, Lely. Hay más textos transcritos ortográficamente desde la oralidad del barranqueño, cfr., en ese sentido (Navas 2014).

${ }^{8}$ Noticia recogida por L. M. Lourenço (09/12/2007).

${ }^{9} \mathrm{El}$ proyecto ha finalizado el diciembre último, desgraciadamente hasta el momento, con pocos resultados.
} 
para que possamos introduzí-lo como disciplina opcional na Escola Básica Integrada de Barrancos".

Pensamos, en definitiva, a partir de los datos recogidos tiempo atrás y ahora dados a la luz, que es necesario, como decíamos, un nuevo acercamiento a la comunidad, donde con seguridad una nueva muestra dará pie a otros resultados, después de las manifestaciones que los barranqueños han venido desarrollando en favor de su lengua, defendiéndola en los foros nacionales y locales en las dos últimas décadas. Porque entendemos que el presente trabajo es un corte temporal sobre las actitudes lingüísticas de los barranqueños que convendría contrastar, pasado este espacio de tiempo, con las nuevas circunstancias, una vez que la comunidad ha manifestado oficialmente la gran autoestima por su lengua.

\section{Referencias bibliográficas}

ADRAGÃO, José Víctor (1976): "Raports locuteur-code: un cas de choix libre?", in Attti XIV Congresso Internazionale di Lingüística e Filologia Romanza, Napoli, 15-20 aprile, 1974. Nápoles: Gaetano Macchiaroli, tomo II, pp. 567-575.

ELIZAINCín, Adolfo (1992): Dialectos en contacto. Español y portugués en España y América. Montevideo: Arca.

FRANCO, Norberto (2000). O porquê de Barrancos: a cultura, a história, os toiros, o Direito. Amareleja: Gráfica Eborense.

LOURENÇO, L. M. (2007): "Município quer tornar barranquenho dialecto oficial e Património Linguístico Nacional” (09/12/2007). Versión electrónica http://www1.rtp.pt/noticias/?article=163602\&visual=3\&layout=10 [consulta 21/10/2010].

NAVAS SÁNCHEZ-ÉLEZ, María Victoria (2011): El barranqueño. Un modelo de lenguas en contacto. Madrid: Editorial Complutense/Centro de Linguística da Universidade de Lisboa.

NAVAS SÁNCHEZ-ÉLEZ, María Victoria (2014): "Presente, pasado y futuro de una lengua de contacto lusoespañola", in Fronteras y diálogos. El español y otras lenguas, J. M. Santos Rovira (ed.), pp. 89-100. Lugo: Axac.

Silva-CoRvalán, Carmen (1988): Sociolingüistica. Teoría y análisis. Madrid: Alhambra Universidad.

VASCONCELOS, José Leite de (1955): Filologia barranquenha. Apontamentos para o seu estudo. Lisboa: Imprensa Nacional. 